\title{
EIN MODERNER LÜGENBARON \\ UND SEIN BIOGRAF. ÜBER MAC ECK'S \\ SONDERBARE REISEN ZWISCHEN KONSTANTINOPEL \\ UND SAN FRANCISCO (1901)
}

\author{
ENDRE HÁRS \\ Institut für Germanistik, Universität Szeged \\ hars@lit.u-szeged.hu
}

\begin{abstract}
Der Wiener Feuilletonist Ludwig Hevesi (1843-1910) hat im Band Mac Eck's Sonderbare Reisen zwischen Konstantinopel und San Francisco (1901) die Reiseerlebnisse des Wiener Fabrikanten und Privatgelehrten Friedrich Eckstein (1861-1939) veröffentlicht bzw. nacherzählt. Die Besonderheit der Sammlung besteht in der doppelten Autorschaft, die sich aus der Verquickung der narrativen Stimmen des mündlich berichtenden Eckstein und des protokollierenden Hevesi ergibt. „Mac Ecks" Berichte über die USA dokumentieren den touristischen Erfahrungshorizont der Gründerzeit und die Auseinandersetzung Wiener Intellektueller mit dem Amerikanismus.
\end{abstract}

Schlagwörter: Reisefeuilleton, Gründerzeit, Moderne, Autorschaft, Amerikanismus

Das Lebenswerk des Wiener Feuilletonisten Ludwig Hevesi (1843-1910) umfasst zahlreiche Schriften und mehrere Genres, mit denen sich das schriftstellerische Profil eines um 1900 tätigen Publizisten und Intellektuellen charakterisieren lässt. ${ }^{1}$ Der hohe Anteil von Reiseberichten in diesem Korpus dokumentiert nicht nur den Lebensstil des liberalen Bürgertums des ausgehenden 19. Jahrhunderts, sondern auch Hevesis Reiseleidenschaft. Der Feuilletonist hat zum einen aus kunsthistorischem Interesse, zum anderen aus touristischer Neugierde zahlreiche Länder Europas bereist und über seine Erfahrungen in beobachtungsreichen humoristischen Reiseberichten auch seine Leserschaft fleißig informiert. Über Europa hinaus hat er sich viel weniger orientiert, wobei er dafür, was versäumt wurde, auf besondere, nämlich literarische Art und Weise schadlos zu halten wusste. Nach seinem berühmten frühen Jugendbuch ${ }^{2}$ und einer Biografie ${ }^{3}$, die jeweils Reisen und Abenteuern anderer gewidmet waren, veröffentliche Ludwig Hevesi 1901 erneut eine Sammlung von Reiseskizzen, in denen wieder einmal fremde Reiseerlebnisse ,adaptiert' wurden. Allerdings ist die Beziehung zwischen Erlebnis und Bericht in diesem Fall auch viel komplexer geraten, als in den beiden früheren Werken. Beruhen diese auf historischen Quellen - vergleichbar mit Schriften von bzw. 
über Andreas Jelky und Wilhelm Junker -, so ist Mac Eck's Sonderbare Reisen zwischen Konstantinopel und San Francisco ${ }^{4}$ die Verschriftlichung von mündlich Erzähltem, dessen ,Original ' mit Ausnahme weniger Aufzeichnungen nie vorgelegen hat. Abgesehen von etwa dreißig Seiten Eigenbericht, die sich über Mac Eck's Reisen in Friedrich Ecksteins (1861-1939) Memoiren „Alte unnennbare Tage!“ Erinnerungen aus siebzig Lehr- und Wanderjahren (1936) befinden, ${ }^{5}$ bleibt selbst die Eckstein-Forschung auf Hevesis Buch als Quelle angewiesen. Im folgenden soll das Doppel des erlebenden-erzählenden Protagonisten Eckstein und dessen Protokollisten Hevesi näher untersucht werden. Die in dieser Frage gefällte Entscheidung ermöglicht im Anschluss daran die Analyse des Bandes als Hevesis ,amerikanischem Reisebuch` bzw. als Bestandteil seines CEuvres.

Friedrich Eckstein, Fabrikant, Privatgelehrter und ,Polyhistor', war eine durch sein Netzwerk und seine verschiedenen Professionen bekannte Gestalt Wiens und der späten Monarchiezeit. ${ }^{6}$ Weniger seine philosophisch-esoterischen Schriften als seine Beteiligung am gesellschaftlichen Leben der Hauptstadt haben in Aufzeichnungen und Erinnerungen der Zeitgenossen ihren Niederschlag gefunden. Seine Biografen kehren vor allem Ecksteins Wagnerismus, theosophische Aktivitäten, Freundschaften mit Musikern und Bekanntschaften mit zahlreichen namhaften Intellektuellen seiner Zeit hervor. Anekdotisch verbürgt sind Ecksteins besondere Kenntnisse der zeitgenössischen Wissenschaften und Künste, mit denen er immer wieder zu imponieren wusste. ${ }^{7}$ Als extravagante Figur und fleißiger Mitgestalter des Wiener Kaffeehauslebens wurde er nicht nur durch Hevesi, sondern auch in anderen Texten der Epoche porträtiert. ${ }^{8}$

Die genannten Memoiren des über siebzigjährigen Eckstein setzen bezüglich des Erinnerungswürdigen selbst Akzente. Es handelt sich um keine streng chronologisch gehaltene Autobiografie, sondern um eine Interessengebiete und persönliche Begegnungen gebündelte Folge von Berichten, die in thematische Kapitel unterteilt sind. Sieht man von den knapp gehaltenen Kindheitserinnerungen im ersten Kapitel und dem englisch-amerikanischen Reisebericht in den beiden letzten Kapiteln des Bandes ab, so beschäftigt sich Eckstein vorwiegend mit seinen Bekanntschaften mit berühmten Persönlichkeiten. Eckstein porträtiert systematisch seine ,Helden“ (Beschreibungen des Äußeren nicht ausgenommen) und liefert - statt Zeitgeschichte - Miszellen zur einer modern-individualistischen Intellektuellengeschichte. Die Rhetorik ist durch die Emphase der Wirkung der betreffenden Künstler und Wissenschaftler auf Eckstein bestimmt, nicht ohne die Hervorhebung des eigenen Anteils an deren Schicksal. Ecksteins Selbstbild wird an den Ruhm und den Kult anderer gekoppelt und wirkt insofern auch kompensatorisch. Dennoch wartet die Autobiografie auch mit wertvollen zeithistorischen Details und Anekdoten, vor allem aus dem Fin de Siècle auf. Im vorliegenden Zusammenhang ist von Bedeutung, dass Eckstein Hevesi völlig unerwähnt lässt. Verblüffend ist vor allem, dass Hevesi als Verfasser ,des Lebens' Ecksteins, 
als Biograph des Ruhmsüchtigen komplett unterschlagen wird. Symptomatisch wirkt auch, dass im amerikanischen Reisebericht gar nicht auf Hevesis Buch zurückgegriffen wird. Die Auswahl des Stoffes überschneidet sich zum Teil mit Mac Eck's Sonderbaren Reisen, unterscheidet sich jedoch sowohl im Wortlaut als auch in den gegebenen Informationen. Der Erinnerungskünstler Eckstein scheint hier seine sonst gern erzählten ,amerikanischen Geschichten“9 hintangestellt und dabei mindestens einen wirksamen „Freund“ ${ }^{10}$ restlos verdrängt zu haben.

Der Titel Mac Eck's Sonderbare Reisen zwischen Konstantinopel und San Francisco ist eigentlich irreführend hinsichtlich der im Band beschriebenen Destinationen. Er verspricht zum einen Abenteuer, zum anderen eine Odyssee, wie sie Jelkys Reisen geboten haben. Erwartungen dieser Art werden im Fall Mac Ecks nur zum Teil erfüllt. Das Reisen bleibt in den im Band behandelten ,Lebensphasen' Ecksteins durch deren biographisches Profil bestimmt. Die Zusammenstellung des Bandes folgt nicht der Lebenschronologie. Den Anfang machen Reisebeschreibungen aus Groß-Britannien ${ }^{11}$ sowie Erlebnisberichte aus esoterischen und theosophischen Kreisen daselbst ${ }^{12}$ (Erlebnisse aus der zweiten Hälfte der 1880er-Jahre). Am zahlreichsten sind Reiseberichte über die USA (Erlebnisse der ersten Hälfte der 1890er-Jahre ${ }^{13}$. Den Abschluss bilden die ,Jugendreisen “ im nördlichen und östlichen Europa (Anfang bis Mitte der 1880er-Jahre) ${ }^{14}$. Auf die Datierung der Reisen wird nicht sonderlich Acht gegeben, so dass man nur aus vereinzelten Hinweisen auf den Zeitpunkt der Reise schließen kann. Die Gespräche zwischen Eckstein und Hevesi sind in der zweiten Hälfte der 1890er-Jahre zu verorten, sodass sich zwischen dem Reise- bzw. Entstehungskontext der Texte und Ecksteins Memoiren immerhin mehr als vierzig Jahre Lebenszeit erstrecken.

\section{Das Interviewbuch}

Grund für das unharmonisch anmutende Nachleben von Mac Eck's Sonderbaren Reisen zwischen Konstantinopel und San Francisco in Ecksteins Erinnerungen ist vielleicht gerade die dem Band zugrundeliegende doppelte Autorschaft. Ecksteins Biographin, Mulot-Déri, scheint selbst in Verlegenheit zu sein, wenn sie sich auf den Band beziehen muss. „Eckstein hat diese Reise [nach Griechenland, E.H.] als ,Ein Pfingsttritt durch den Peloponnes' in Ludwig Hevesis Sammelband [...] beschrieben" ${ }^{\text {"15 }}$ - schreibt sie an einer Stelle, Eckstein als eigentlichen Akteur vortreten lassend. Dennoch erkennt sie, wenngleich mit abschätzigen Kommentaren, auch Hevesis schriftstellerischen Beitrag an. Auf das andere Extrem stößt man in zeitgenössischen Kritiken. ,[E]s kann einer lange erzählen, ohne als Erzähler sein Glück zu machen, wenn er nicht einen Zuhörer findet wie Ludwig Hevesi“", schreibt Wilhelm Goldbaum im Pester Lloyd. ${ }^{16}$ Eckstein könne sich glücklich nennen, dass seine ,krause[n] Geschichten sich in kleine Wunder- 
werke humoristischer Reise-Erzählung verwandel[t] " haben und er dadurch „,auf einem Umwege seinen Einzug in die Literatur gehalten hat" ${ }^{\star 17}$. Noch fragwürdiger wird Ecksteins Rolle bei einem Rezensenten der Wiener Zeitung, der - Hevesis Vorwort ignorierend - gar erst seine Dilemmata artikuliert, wie denn überhaupt Hevesi an diesen Stoff gelangt sei und ein derart „anglo-amerikanisches“ Buch „in Wien schreiben konnte" ${ }^{18}$.

Über die urheberische „Doppelgestalt" ${ }^{\star 19}$ äußert sich jedenfalls auch Hevesi selbstbewusst und seinem Medium gegenüber dennoch die gebührende Ehre erweisend. Er hält in seinem Vorwort fest, dass er von Ecksteins „Reisen, von denen [dies]er [...] wie ein Odysseus bunt zu erzählen pflegte“ (Vo VI), ,halb in seiner, halb in meiner Weise" (ebd.) Aufzeichnungen zu machen angefangen hat. Dabei vermerkt er auch: „Die Darstellungsform als zusammenhängende Erzählung ergab sich von selbst, obgleich die mündliche Mitteilungsweise je nach Stimmung und zufälliger Gedankenverbindung zwangloser war und mich auch aus eigenem beträchtlich mitwirken ließ.“ (ebd.) Auch Hevesi hält sich also selbst für mehr als für einen bloßen Protokollisten, und charakterisiert die Zusammenarbeit nochmal, als hätte man dabei „vierhändig Klavier gespielt“ (Vo VII). Er findet auch für die Perspektive des erzählenden Ichs der Texte eine treffende Beschreibung: Die „technisch-sportlichen Augen“ Ecksteins seien ganz andere gewesen, als seine eigenen „litterarisch-künstlerischen“, ,,aber ich hatte sie gewissermaßen in meinem eigenen Kopf" (Vo VI-VII). Ecksteins Bericht sei insofern keine Zitation, sondern schriftstellerische Leistung, in deren Rahmen letztendlich Hevesi als Erzähler und Eckstein als Figur agieren.

Die Differenz zwischen dem berichtenden und dem protokollierenden Ich ist in den Beiträgen mehrfach markiert. Die Vorlage bildet das Modell des Interviews als Abschrift bzw. nachträgliche Redaktion eines Gesprächs. Hevesi belässt es nicht beim Vorwort, sondern schreibt sich als Herausgeber in den Text mit ein. Die Hauptregel für die Herstellung der erforderlichen Referenz ist in den einzelnen Beiträgen eine kurze Formel zum Auftakt des Textes, die, als Parenthese nach dem Muster , sagte Freund Fritz', in die beginnende Ich-Erzählung eingeschoben wird. ${ }^{20}$ Dieses Signal hat auch in den Zeitungsveröffentlichungen - die gleichwohl öfter auch mit Untertiteln und Anmerkungen versehen wurden ${ }^{21}$ - den Kontext mit früheren Mitteilungen dieser Art hergestellt. Die Pflichtformel, sagte Freund Fritz' wird in den Texten durch weitere Hinweise auf den berichtenden Freund und dessen Stil ergänzt, spricht doch „Fritz“ seinen Gesprächspartner immer wieder an, und fordert ihn mit Floskeln wie ,Sie wissen, dass ${ }^{622}$ wiederholt heraus. Diese Formeln sind das konsequent verwendete Minimum, sonst fallen Ecksteins Erzählung und deren Wiedergabe zusammen, als würde man unmittelbar Eckstein lesen bzw. reden hören.

Am geringsten fiktionalisiert ist die Beziehung zwischen dem berichtenden und dem protokollierenden Ich in drei Fällen: In Eine Minute mit Edison ist Eck- 
steins Text durch Anführungszeichen von den (spärlichen) Erzählerkommentaren Hevesis abgesetzt; in Jack Strap und der Drummer erzählt Hevesi über die Erlebnisse seines Freundes in dritter Person Singular; schließlich ist dem Beitrag Jostedalsbrä eine längere Einführung über Eckstein in dritter Person Singular vorangestellt. ${ }^{23}$ In diesen Fällen werden die Zuständigkeiten jeweils dem einen oder anderen Part zugeordnet und der Charakter des Interviewbuchs hervorgekehrt. Es gibt aber zahlreiche Fälle, in denen die Markierung der Differenz den umgekehrten Effekt erzielt. Die Textanfänge thematisieren zwar den Gesprächsrahmen, womöglich auch die Umstände des Interviews, das sogar durch Verabredung und Besuch arrangiert wurde. Aber man liest über all das in Form einer Rahmenerzählung. Der durchstilisierte Bericht darüber, wie das Buchprojekt entstanden ist, wird selbst zu einem Narrativ, in das Ecksteins Berichte als Geschichten des Ich-Erzählers „Mac Eck“ eingebettet werden. Charakteristisch sind diesbezüglich z. B. folgende Stellen:

Also von Madame Blavatsky soll ich Ihnen erzählen? Sagte Freund

Fritz und zog die Augenbrauen ungewöhnlich hoch. (MB 15) ${ }^{24}$

Ich bin heute zu müde, mit Ihnen im Geiste nach Amerika zu gehen sagte Freund Fritz -, bleiben wir einmal unterwegs liegen. Im östlichen London vielleicht, wo es auch sehr sonderbar zugehen kann.“"(FG 59)

„Was das Abspringen von fahrenden Bahnzügen betrifft, so [...] gehört eine eigene Turntechnik dazu. (Der Erzähler demonstrierte sie sogleich; es muß übrigens bemerkt werden, daß er von Kindesbeinen auf in allen Leibesübungen Champion ist.) “(EA 102) ${ }^{25}$

In diesen Szenen gewinnt Eckstein als (Erzähler-)Figur Gestalt und die Umstände der Begegnung werden selbst zum Erzählstoff. Ecksteins sprachliche Eigenarten, z.B. seine im Text wiedergegebenen erzählerischen Digressionen, werden durch einen von außen auf ihn geworfenen Blick ergänzt. Die Rahmenerzählung hat aber auch für den Protokollisten Konsequenzen - konstituiert ihn doch dieser Rahmen erst als Ich-Erzähler. Auf dieser Ebene sind die Formeln , sagte Freund Fritz' verortet, und je mehr dazu gesagt wird, desto mehr wird die scheinbar beiläufige Befragung selbst zum Handlungsstrang. Hevesi selbst wird zur Figur, die eigene Beobachtungen macht und von „Fritz“ mit ins Gespräch gezogen wird:

"[...] Sie wissen, was ein Teller ist?"

„Gewiß,“ sagte ich, „eine kleine Schüssel...“

„Ach, Sie sind ein Grünhorn!“ rief er aus. „Ich meine ja keinen Teller, sondern einen teller: von to tell, sagen; also einen Ansager gleichsam.

$[\ldots](\mathrm{ME} 123)^{26}$ 
Der hydraulische Druck führt hier das große Wort. Wissen Sie, was Druck ist? ... Ach, Sie wollen sagen, daß Sie selbst für den Druck arbeiten? Nun, kommen Sie einmal zu mir und ich will vor Ihren Augen Ihr letztes Buch, „Blaue Fernen“ glaube ich, das fünf Centimeter dick ist, in eine durchsichtige, unzerbrechliche Glasplatte von 2 Millimeter Stärke verwandeln. Ich übergieße es bloß mit einer Säure und setze es einem hydraulischen Druck aus, der nicht von Pappe ist. (ME 132) ${ }^{27}$

Wie letzteres Zitat belegt, geht es hier sehr wohl um die reale Person Ludwig Hevesi und dessen Partner, den Fabrikanten Friedrich Eckstein. Die Anlehnung an die eigenen Lebens- und Reiseerfahrungen ändert aber nichts daran, dass deren Verschriftlichung anders verläuft, als wenn Eckstein seine Erinnerungen selbst verfasst hätte. Durch Überantwortung des Erzählens ändert sich der Status des berichtenden Ichs, dessen Erfahrungen erst durch das protokollierende Ich zum Text werden. Die narrative Instanz ersten Grades ist notgedrungen Hevesi, und diesem steht es durchaus frei, durch Ausbau der Rahmenerzählung auch sich selbst mit ins Spiel zu bringen. Zudringlich und übertrieben kann diese narrative Selbstverwirklichung nicht genannt werden, dennoch dürfte sie zur Verdrängung des Bandes in Ecksteins späteren Memoiren beigetragen haben.

Hevesi nimmt seine Möglichkeiten als vermittelnde narrative Instanz immer wieder wahr, so dass die erzählerische Besonderheit des Bandes nie aus den Augen gerät und dem Reisebericht ein besonderes Gepräge verleiht. ${ }^{28}$ Die narrative Ordnung der doppelten Autorschaft wird vielleicht am ausdrücklichsten im Beitrag Im Rauchsalon selbstreflexiv gewendet. Hier legt Hevesi auf die Stilisierung des Erzählstils von Eckstein besonderen Wert. Bereits der Auftakt des Textes nimmt explizit auf die Situation Bezug:

\footnotetext{
„Sofort - sagte Freund Fritz - ich will nur erst diese Flasche Goldeck entkorken. (Er zog einen blinkenden Gegenstand aus der Tasche.) Sehen Sie, das kriegt man in den ganzen Vereinigten Staaten auf der Straße geschenkt. [...] Auf der Hülse steht nichts zu lesen, als der eingravierte Name Anhäuser-Busch. Das genügt. Anhäuser-Busch ist nämlich die Riesenbrauerei in Saint-Louis (Missouri). Sie wissen, eine Brauerei, so groß wie eine Provinzialhauptstadt.“ (Ra 73)
}

Schon dieser Anfang deutet an, wie wenig sich der Berichterstatter an den Hauptstrang seiner Erzählung halten wird. Entsprechend wiederholt sich im Text fünfmal die Wendung ,Doch um zur Sache zu kommen', sodass der ganze Beitrag durch Digressionen strukturiert wird. Hierzu passt auch der Inhalt des Textes: Eckstein erzählt, wie die Passagiere der Transatlantikschiffe die Zeit vertreiben. Neben „Poker und Whiskey (Ra 75) sind dabei vor allem „Wetten und Anekdotenerzählen“ (ebd.) von Bedeutung. Ecksteins Erzählung vergegenwärtigt, wie 
sich die von Reisenden präsentierten Geschichten einander ohne System und Ordnung durchkreuzen:

Sie reißen sich gegenseitig das Wort aus dem Munde. Einer sucht
dem andern zuvorzukommen, indem er, bevor noch sein Vordermann
geendet hat, bereits beginnt: „Da wir gerade von Buffalo sprechen,
fällt mir ein“ u.s.w. In schleppendem, nachdrücklichem Tone beginnt
er, noch unverständlich im Gelächter über die Pointe der vorigen
Geschichte, aber er hat den Faden erwischt und den Anfang des
seinigen in das Ende des vorigen „hineingetwistet“. [...]
Doch um zu den Anekdoten zu kommen...“ (Ra 77)

Die Schilderung dieser Szene wird zur Mise en abyme der Gesprächssituation zwischen Hevesi und Eckstein. Dieser überbietet zwar nur sich selbst im Geschichtenerzählen, tut es jedenfalls nicht anders als die geschilderten „Drummers". Die zitierte Stelle ist eine Anekdote, deren Sujet auch Ecksteins chaotisches Erzählen charakterisiert. Dieses wird freilich als durchkomponiertes Chaos des, wortgetreuen' Protokollisten wiedergegeben. Während der Überblick über die eigene erzählerische Leistung „Mac Eck“ selbst verwehrt bleibt, setzt Erzähler Hevesi den Leser in die Lage, die Parallelen zu erkennen. Das hintergründige Porträt Ecksteins als Erzähler ist ein Zusatz, der durch die Rahmenerzählung ermöglicht wird und Hevesi zum Karikaturisten eines Chaoten werden lässt. ${ }^{29} \mathrm{Im}$ Dienste des anderen kommt auch er selbst auf seine (feuilletonistische) Rechnung. Muss er doch auch selbst um ergiebige Unterhaltung der Konsumenten seiner Geschichten über Eckstein sorgen. Und da ist mehr Witz (als Eckstein erzählen wollte) besser als weniger.

Als ein weiterer, das gesamte Bandkonzept charakterisierender Beitrag kann schließlich der Bericht Nach Klondyke betrachtet werden. Auch hier handelt es sich um eine Verschachtelung von Erzählungen: „Nun thut es mir leid, daß ich in San Francisco keine Zeit mehr hatte, den wochenlangen Abstecher nach Alaska zu machen“" (NK 274) - sagt Freund Fritz und nimmt diese Beteuerung zum Anlass, aus vor ihm liegenden ,press-cuttings“ (NK 276) die Erfahrungen eines Goldgräbers in Alaska zu rekapitulieren. Eckstein, der eine solche ,Reise“ nicht gemacht hat - wenngleich es seinem Ehrgeiz sehr entsprochen hätte -, berichtet über sie aus anderweitigen Quellen und kann sich dabei auch auf zwei Freunde beziehen, die es immerhin - wenngleich erfolglos - versucht haben. Eckstein war zwar nie da, habe jedoch gerade mit diesen Freunden „eine halbe Stunde von Wien [...] gewisse Wände“ erobert, ,die schon berühmte Ortler- und Matterhornführer ,paff" gemacht haben“ (NK 276). Sie haben Bergrücken zusammen erstiegen, die, wenngleich nicht gleich in Alaska, so doch auch bei Wien selbst sehr schwer zu schaffen waren. Einerseits muss man also zum Bestehen schwieriger Proben nicht gleich ans Ende der Welt reisen, andererseits kann man 
für sich Freunde einspringen lassen, deren Erfahrungen ebenso übertragbar, wie wilde Orte austauschbar sind. Eckstein, der seine sportlichen Leistungen durchgehend stolz hervorhebt, erreicht hier Alaska gleichsam durch ,Fernsteuerung'. Und während die genannten press-cuttings der Zeitungen das Goldgräberunternehmen als etwas Machbares und Leichtes beschreiben, weiß der berichtende Eckstein nun aus Erfahrung - aus der seiner Freunde bzw. aus Erlebnissen, die bei Wien gemacht wurden - ganz genau, wie beschwerlich ein solches Unternehmen ist. Damit kehrt der Beitrag hervor, was auch an anderen Stellen vorsichtig angedeutet wird, dass „Mac Eck“ ein Erzähler sei, bei dem es nicht auf das letzte Faktum ankommt. Sein Selbstbild ist stark narrativ besetzt und nur mit Abstrichen für eine Biografie geeignet. Durch diese Erkenntnis rückt wieder der Rahmenerzähler Hevesi ins Bild, der dies erkannt hat und in diesem Sinne „Mac Eck"statt Friedrich Eckstein zu verewigen weiss.

\section{Reise in die Moderne}

Die doppelte Autorschaft in Mac Eck's Sonderbaren Reisen zwischen Konstantinopel und San Francisco verschafft dem Band einen besonderen Status im Hevesi CEuvre. Die Thematik verankert ihn unter den Reisebüchern des Feuilletonisten, und die Tatsache, dass dessen Grundlage ,fremde' Reisen bilden, unterscheidet ihn wieder von Ihnen. Dennoch sind die Akzente anders gesetzt, als etwa im Buch über Wilhelm Junker oder in Hevesis anderweitigen Biografien. ${ }^{30}$ Hier erzählt die porträtierte Person selbst, was zum einen die oben beschriebene Literarisierung als Figur zur Folge hat, zum anderen den Gegenstand seiner Erzählung hervorkehrt: Die Reiseerlebnisse stehen im Vordergrund, sie entsprechen dem Impetus der Reiseliteratur der Zeit ${ }^{31}$ und verorten das Werk statt der Biografien im Kontext von Hevesis Reisebüchern. Bei deren großer Vielfalt fällt hier auf, dass Hevesi mit Hilfe des Mediums Mac Eck nicht nur eine Reise ,nachholt ${ }^{6}$, die er persönlich nie gemacht hat, sondern Themen aufgreift, mit denen er höchstens im westlichen Europa in Berührung gekommen ist.

Mac Eck's Sonderbare Reisen zwischen Konstantinopel und San Francisco greift drei große Themen der Zeit auf: Amerika, den Esoterismus und den Alpinismus (Berg- und Extremtourismus). Während die beiden letzten Themen in nur jeweils drei Beiträgen behandelt werden, widmen sich vierzehn Texte Reisen in den USA, wobei von ihrem Thema her auch die beiden britischen Geschichten zu dieser Gruppe gerechnet werden können: In diesen Texten reist der durchaus technisch interessierte „Fritz“ in Länder der Modernisierung und erkundet eine für mitteleuropäische Verhältnisse fortschrittliche ,neue Welt'. Dabei bedient er sich der Stereotype und der Klischees des in diesem Zeitraum gängigen Amerika-Diskurses: Die Reise führt statt aus der modernen Welt hinaus mitten in sie 
hinein, ${ }^{32}$ bei mal distanzierten, mal affirmativen Beobachtungen der für Europäer verblüffenden Extreme.

Vergleicht man den Ansatz mit der zeitgenössischen Reiseliteratur über die USA, so ergeben sich zahlreiche Parallelen. Eckstein bereist die nördliche Ostküste (New York, Philadelphia, Boston, Newport, Albany), besucht Wisconsin (Milwaukee), Illinois (Chicago), Indiana (Indianapolis), Kentucky (Louisville), Missouri (St. Louis) und reist auch in den Westen, nach Colorado (Denver, Colorado Springs, Grand Junction), Utah (Salt Lake City) und Kalifornien (San Francisco). Damit absolviert er Reiserouten, die um diese Zeit die durchschnittlichen Ziele der Europäer waren. ${ }^{33}$ Er gehört zu den wohlhabenden Amerikareisenden, die sich die Reise auf dem Oberdeck der Kreuzfahrtschiffe und die kostspieligen Zugfahrten und Luxuswaggons leisten können. ${ }^{34}$ Er besucht auch teure Orte (z.B. die Bade- und Kurorte Newport, Rhode Island, Saratoga Springs, Colorado Springs ${ }^{35}$ ) und trifft sich mit der gesellschaftlichen Elite. ${ }^{36}$ Dieses Profil verstärken zahlreiche Themen ,Mac Ecks“: Beschreibungen des Hotelwesens, ${ }^{37}$ Berichte über die Infrastruktur der Städte und des Landes, über Menschen und Gewohnheiten. Man beobachtet dabei eine starke Tendenz zu den amerikanischen Besonderheiten. Der Erzähler konfrontiert mit „österreich-ungarischem Erstaunen“ $(\text { Ki 211) })^{38}$ „kontinentale ${ }^{639}$ Erwartungen mit überseeischen Überraschungen, und beschreibt die „Yankee[s]“ (JS 119) als innovativ, reich ${ }^{40}$ und exzentrisch:

\footnotetext{
„Wissen Sie zum Beispiel, wie man es angefangen hat, sämtliche Ausstellungsgebäude [der Weltausstellung in Chicago, E. H.] schneeweiß zu lackieren? Man stellte den weißen Lack in ungeheuren Bassins auf und sprühte ihn mittels Dampfspritzen über alle die großen und kleinen Bauwerke. [...] Und ein guter Spaß war es auch, was man that, um sich zuletzt die kostspieligen Abtragungsarbeiten zu ersparen. Man stecke einfach die ganze Geschichte in Brand und ließ sie zu Asche werden, aber wohlgemerkt, gegen Eintrittsgeld. (EC 165)

„Der alte Hin- und Hertraber [ein Museumswächter, E.] erzählte mir dabei von einer Wette, die Mr. Astor, einer von der gewissen Achtnullenfamilie, in London mit Lord Soundso eingegangen sei. Nämlich 250 Personen an einem Tische zu bewirten, der aus einer einzigen Platte [dem Querschnitt eines Riesenbaums, E. H.] bestehen werde. [...] Er gewann die Wette. Die amerikanischen Zeitungen aber waren wütend über Mr. Astor, weil er so ,schäbig' gewesen, eine so kleine Sequoja [Sequoja gigantea, E. H.] zu kaufen und die Reputation der Vereinigten Staaten in England nicht besser zu verteidigen [...].“ $(\mathrm{SF} 262)^{41}$
}

„Mac Eck“ beobachtet kulturelle Spezifika, nationale und regionale Klischees. Er kommentiert immer wieder das Großstädtische, ${ }^{42}$ die amerikanische „Kriminalromantik“ (SM 148) ${ }^{43}$, den „Kentuckysmus“ (Ke 187) ${ }^{44}$, den Mormonismus ${ }^{45}$ 
und beschreibt viele ,Kuriosa': die Mustangs (Ke 195), das Popcorn (Ke 198), den Kautabak (Ke 185), den Kaugummi (SD 156), das Müsli (eigentlich das „Shucotash“ der „Rothäute“, SM 150), den „Maple-room“446 und das „Preistöten“ (SF 270, im Sinne von ,Ausverkauf'). Er , begegnet' der , amerikanischen Mentalität' und dem Nationalbewusstsein, entzifferbar etwa in ,Bekenntnissen“ zum Amerikanertum von der Art: „Sie wollten keine englischen Lords werden, sondern amerikanische Bürger bleiben und das Yacht-Bootsgeschäft weiter treiben." (SM 145) Die nachdrücklichste Erfahrung ist für ihn - als dezidiert technisch interessierten Europäer - jedoch die Modernisierung. „Die Amerikaner müssen recht haben“, heißt es in Kikapu, ,wenn sie behaupten, ihre Luft sei weit elektrischer als irgend eine andere, und je weiter nach Westen, desto elektrischer.“ (Ki 213). Der Satz ist zwar konkret gemeint, ist aber symptomatisch für Ecksteins Eindrücke: In den Vereinigten Staaten liege die technische Innovation gleichsam in der Luft und beherrsche alles. ${ }^{47}$,[W]er könnte sich in Amerika langweilen“(EC 178), vermerkt er auf einer längeren Fahrt auf der Michigan-See, wenn schon das Schiff an sich - ein „Walfischdampfer“ (ebd.) - etwas Besonderes ist, und man langweilt sich gar erst nicht, wo an Bord regelrechte „Bicycle-Rennen“" (ebd.) organisiert werden. Kein Wunder, wenn die Erzählung auch dann bei diesen Themen ankommt, wenn sie dem vermeintlichen Interesse des Gesprächspartners Hevesi bzw. der Leserschaft entgegenlaufen:

\footnotetext{
„Was mir in Salt Lake City am besten gefallen hat? [...] [E]s war die Feuerwehr ... Das scheint Ihnen nicht recht zu sein, aber ich kann Ihnen nicht helfen, sie hat mir eben außerordentlich imponiert." (BM 226)

„Doch ... wollt‘ ich Ihnen nicht von der Feuerwehr erzählen? Ja, das ist das Schönste in Salt Lake City. Aber Sie verstehen ja ohnehin nichts davon."(BM 241).
}

Versucht man das Spezifische von Mac Eck's Sonderbaren Reisen zwischen Konstantinopel und San Francisco hervorzukehren, so sind zunächst einmal seine thematischen Schwerpunkte zu nennen. Eckstein ist zwar in zahlreichen Lebensbereichen bewandert, beruflich ist er aber ein Chemiker und Fabrikant, dessen Reisen zum Teil auch fachspezifische Gründe haben. Die Geschichte der Begegnung mit Edison [im Beitrag Eine Minute mit Edison] wird durch ausführliche Beschreibung der organisatorischen Details eingeleitet, die nötig waren, um überhaupt nach Schenectady fahren zu dürfen, und sie bietet die Beschreibung der Anreise und des Fabrikgeländes der damaligen General Electric Company, so dass der Text den langen Weg und die kurze Begegnung auch rhetorisch ausspielt. Nachdem Edison Eckstein mit einem Händeschütteln und wenigen Sätzen abgefertigt hat, tröstet sein Begleiter, Mr. Green, den enttäuschten ,Pilger` mit den Worten: „O, eine Mi- 
nute ist sehr viel, Sie dürfen ganz zufrieden sein" (ME 135), wodurch einmal mehr das Klischee der amerikanischen Geschäftigkeit bestätigt wird.

Zum spezifischen Blick des Erzählers bedarf es jedoch nicht zwingend des technischen Umfeldes. Auch sonst richtet sich seine Aufmerksamkeit immer wieder auf derlei Momente. „Ich bin Barbar und bewunderte natürlich den Maschinentempel“ (BM 232), sagt er über sich anlässlich des Berichts über Salt Lake City. Diesem Anspruch (einer Art gespielter Anspruchslosigkeit) folgend unterlässt er nie, die benutzten Verkehrsmittel zu beschreiben, ${ }^{48}$ die Architektur auf ihre Funktion hin zu kommentieren und generell - in europäisch-amerikanischer Gegenüberstellung - die zivilisatorischen Fortschritte (statt der kulturellen) hervorzuheben. Unausgesprochen vermittelt „Mac Eck“ - denn theoretisch wird er nie - das Bild der USA als einer dezidiert materiellen Kultur, ohne freilich ein negatives Urteil zu fällen. Umgekehrt, er versäumt nicht, das Beobachtete als etwas Besonderes - und Erzählwürdiges - zu präsentieren. So etwa in der Beschreibung des Fährbootes von New York nach Albany:

\footnotetext{
Ueberall eiserne Säulen, Mahagoni und roter Sammt. Als ich dann essen wollte, hieß es: »Sie müssen hinuntergehen! « Ich ging hinunter, in eine zweite solche Halle [...]. Auf meine Frage nach dem Speisesaal hieß es wieder: »Sie müssen hinuntergehen! « Noch ein Stockwerk tiefer speiste ich vortrefflich und steckte mir dann eine Zigarette an. Da kam ein Steward und ersuchte mich nicht zu rauchen. »Dazu müssen Sie hinuntergehen.« In den Rauchsalon nämlich. Ich rauchte um vier Stockwerke tiefer, als ich schlief. (ME 126)
}

Ein weiteres Spezifikum „Mac Ecks“ neben der Vorliebe für die Technik ist sein Interesse an Sportarten und sein Hang zur Sportlichkeit. In diesem Kontext gewinnen die im vorliegenden Rahmen unberücksichtigten norwegischen Geschichten (Jostedalsbrä, Wanderungen in Jotunheim) und das abschließende - hauptsächlich in der Nähe von Graz spielende - Wie man das Abstürzen lernt an Bedeutung. Sie sind alle Berichte über ,Extremsport' um 1900. Aber auch die USA-Feuilletons bringen immer wieder Beobachtungen über den amerikanischen Sport $^{49}$ und Geschichten über Ecksteins eigene ,sportliche Leistungen“, etwa über Sprünge von fahrenden Zügen ${ }^{50}$ und über Kletterabenteuer. ${ }^{51}$

\section{Doppelte Autorschaft}

Mit Ecksteins thematischen Schwerpunkten wäre jedoch weder das Besondere von Mac Eck's Sonderbaren Reisen zwischen Konstantinopel und San Francisco erschöpft noch deren ,Reiz' erklärt. Zum Gesamtbild müssen wieder die narrativen Aspekte aufgenommen und das Gesamkonzept des Bandes berührt wer- 
den. Spezifisch ist die Zusammenstellung bereits dadurch, dass die Sammlung über die Amerika-Berichte hinaus auch andere Reiseberichte enthält, so dass die erlebende-berichtende Person und nicht deren Destinationen den gemeinsamen Nenner bieten. Hauptattraktion ist der Abenteurer, ohne dass im Gesamttext das Autobiografische überhand nehmen würde. Der Reisebericht-Charakter wird auch dadurch relativiert, dass „Mac Eck“ - auf für Hevesi gar nicht ungewöhnliche Weise - immer wieder anti-touristische Anmerkungen macht und sich vom Baedeker-Stil distanziert:

„Dieses Paradestück des Globus [die Mammuthöhle in Kentucky] ist bereits xbis y-mal beschrieben worden, und ich möchte lieber kein Wort darüber verlieren, aber ich habe dort zufällig auch Neues erlebt.“ (Ke 196)

„Wünschen Sie auch, daß ich Ihnen das Tabernakel [am Temple Square in Salt Lake City] schildere? Nicht! Das habe ich von Ihrer Intelligenz und Belesenheit vorausgesetzt. Ich bemerke nur, daß die ungeheure ovale Kuppel auf ihrer Reihe weißer Pfeiler mir [...] wie ein $250 \mathrm{Fuß}$ langer Käfer [erschien][...].“ (BM 232) ${ }^{52}$

In diesen Fällen der Distanzierung fällt sogleich auf, dass „Mac Eck“ bei aller abwehrenden Geste immer unbeirrt weitererzählt. Was er zum Besten gibt, unterscheidet sich jedoch wesentlich vom Mainstream der Reiseliteratur. Ihm kommt es offensichtlich nicht auf Informationen an, die von einer Bildungsreise zeugen oder (Nicht-)Reisende versorgen würden. Statt dessen steht das ,Storytelling “im Mittelpunkt, der Redefluss eines ,Großmauls', eines modernen ,Lügenbarons', in dese Geschichten nach wie vor die Übertreibung und das anekdotische Element vorherrschen. Die Länder, die er besucht, bieten für ihn nicht nur Besonderes und Abenteuerliches, sie bieten etwas, was nur ihm, genau so und nicht anders, hat widerfahren können. So werden in seiner Darstellung, um nur ein Doppel-Beispiel heranzuziehen, überfüllte Eisenbahnwaggons zu Schauplätzen mythisch erhöhter (sexistischer bzw. rassistischer) Menschenschlachten:

Ich bin da einmal hineingeraten und spüre es noch hier ... und hier, besonders wenn sich das Wetter ändern will. [...] Man muß einmal mitten in diesem Publikum gesteckt haben, wenn es im Laufschritt die Remise stürmt und die Waggons besetzt. Aber Sie wissen ja, ich war einst Athlet und weiche noch jetzt nicht leicht einem Ellenbogenduell aus. [...] Als Wagentritte benützte er [ein Schwarzer, der am Erzähler aufs Wagendach klettert, E. H.] wiederum meine Schultern. Nun, er hätte auch, als er oben saß, meinen Kopf als Fußbank verwerten können. Als die Nachlaufenden seinen Erfolg sahen, begannen sie ihm das Akrobatenstück nachzumachen. Es entspann sich eine große Voltige über den Hinterpuffer und meine beiden Schultern hinan, auf das Wagendach. Betrunkene Miners (Grubenarbeiter), Cowboys mit langen Rädersporen an den Fersen, Mulatten, Neger, Irokesen und sogar zwei Damen schwangen sich so an mir empor. (EC 167-168) 
„Mac Eck“ teilt auf diese Art und Weise seine Abenteuer und kuriose Geschichten mit, aber aus den Zeilen zwinkert dabei auch deren Übermittler, der ,Stilist" Hevesi hervor. Je mehr es „Mac Eck“ auf das Geschichtenerzählen ankommt, desto wichtiger wird die Präsentation bzw. die Redaktion seines Textes. Von den beiden Ich-Erzählern ist es eigentlich Hevesi, der sich aufs Humoristische versteht. Die Beziehung und die Rollenverteilung beider relativiert sich und stellt sich in der Ausarbeitung gleichsam auf den Kopf: Das wiedergegebene Interview erweckt zuerst den Eindruck, als wäre es Eckstein, der sich eines Zuhörers und ,Ghostwriters` bedient. Seine Geschichten erweisen ihn hingegen als einen Gesprächspartner, dem Hevesi nicht umsonst so viel Aufmerksamkeit schenkt. Aus Hevesis Perspektive ist Ecksteins Lebensgeschichte verwertbarer Stoff für ein humoristisches Reisebuch. Mögen Amerika, Großbritannien, das nördliche bzw. das südliche Europa mit noch so viel Sensation aufwarten, ,rund' und interessant werden Mac Eck's Sonderbare Reisen zwischen Konstantinopel und San Francisco so recht erst durch die erlebende und erzählende Figur. Diese mitzugestalten liegt allerdings in der Zuständigkeit Hevesis, der auf der Ebene des dargestellten Interviews zwar nur als Sekundant agiert, auf der Ebene der Verschriftlichung jedoch zum Erzähler und Autor avanciert. Insofern bildet die Beziehung der beiden Erzähler zueinander eine Art Kippfigur. Keine Autorschaft Hevesis ohne Eckstein und keine Lebensgeschichte Ecksteins ohne Hevesi. Diese Interdependenz macht den Reiz der Feuilletonsammlung aus und sorgt für deren narrative Spannungskraft. Auf Ecksteins Geschichten legt sich der Schleier einer ,fremden“ erzählerischen Macht und macht sie erst zu dem, was sie sind.

Man kann nur spekulieren, in welchem Maße die doppelte Autorschaft zur Verdrängung beim alten Eckstein geführt hat. Die fehlende Ernsthaftigkeit mag in zweifachem Sinne Stein des Anstoßes gewesen sein. Ecksteins Autobiografie belegt, dass die Reisen nur soweit von Interesse sind, als man berühmte Persönlichkeiten (Edison, Mark Twain) kennenlernen kann. Der Rest passt nicht richtig ins Konzept des kultisch operierenden Zeitzeugen. Amerika ist im Grunde zu modern und zu belanglos im Vergleich zu all den klassischen - künstlerischen, vor allem komponistischen - Werten, die sich im Wien von damals dokumentieren lassen. Darüber hinaus mag sich der alte Eckstein auch damit nicht identifiziert haben, sein Leben als einen Witz, ein Spektakel eines kuriosen Reisenden wiederzusehen. Das Angebertum nimmt in „, Alte unnennbare Tage! “Erinnerungen aus siebzig Lehr- und Wanderjahren noch weiter zu und duldet keine Karikatur. Die - freilich nur vermutbare - Distanzierung ermächtigt freilich einmal mehr zur ,Rehabilitierung‘ des Bandes in bzw. als Hevesis Werk. 


\section{Fußnoten}

1 Sármány-Parsons, Ilona: Ludwig Hevesi als Schöpfer des Kanons der österreichischen Malerei. In: Ders./Szabó, Csaba (Hg.): Ludwig Hevesi und seine Zeit. Wien: Institut für Ungarische Geschichtsforschung in Wien 2015 (= Publikationen der ungarischen Geschichtsforschung in Wien XI), S. 47-70.

2 Hevesi, Lajos: Jelky András bajai fiú rendkívüli kalandjai ötödfél világrészben. Történeti kútforrások alapján a serdültebb ifjúság számára. Pest: Heckenast 1872; Onkel Tom: Des Schneidergesellen Andreas Jelky Abenteuer in vier Welttheilen. Nach historischen Quellen zum ersten Male ausführlich dargestellt und der reiferen Jugend gewidmet. Budapest: Franklin 1875.

3 Hevesi, Ludwig: Wilhelm Junker. Lebensbild eines Afrikaforschers. Berlin: Weidmannsche Buchhandlung 1896.

4 Hevesi, Ludwig: Mac Eck's Sonderbare Reisen zwischen Konstantinopel und San Francisco. Stuttgart: Verlag von Adolf Bonz \& Comp. 1901. Im Weiteren mit der Sigle „McE“. Auf Angaben zur Erstveröffentlichung der Feuilletons wird aus Raumgründen verzichtet.

5 Vgl. Eckstein, Friedrich: „Alte unnennbare Tage!“ Erinnerungen aus siebzig Lehr- und Wanderjahren. Wien/Leipzig/Zürich: Herbert Reichner Verlag 1936. Hier zitiert aus der Ausgabe Edition Atelier 1992, S. 253-292.

6 https://anthrowiki.at/Friedrich_Eckstein [19.05.2018];

7 Mehrere Beispiele zitiert: Schönherr, Max: Wer war Friedrich Eckstein? In: Eckstein: „Alte unnennbare Tage!", S. 311-328, hier S. 315-316.

8 Er sei der in Das Unbehagen in der Kultur (1930) erwähnte „Freund”, der Freud über Yogapraktiken berichtet. Vgl. Mulot-Déri, Sibylle: Alte ungenannte Tage. Zu einer Biographie Friedrich Ecksteins. In: Eckstein: „,Alte unnennbare Tage!“, S. 295-310, hier S. 302.

9 Vgl. Schnitzler, Arthur: Tagebuch 1893-1902. Hg. v. M. Werner Welzig. Wien: Verlag der Österreichischen Akademie der Wissenschaften 1989, S. 103 (Eintrag v. 03.12.1894).

10 Hevesi spricht dagegen mehrfach von „mein[em] Freund Fritz Eckstein“. Hevesi, Ludwig: Vorwort. In: McE, S. V-VII, hier S. V. (Sigle „Vo“).

11 L. H-i.: Ein gefahrvoller Nachmittag. In: McE, S. 1-14. (Sigle „GN“); In Forest Gate. In: McE, S. 59-72. (Sigle „FG“).

12 Ein mystischer Besuch. In: McE, S. 15-29 (Sigle „MB“); Mystische Erlebnisse. In: McE, S. 30-47 (Sigle „Er“); Ein mystisches Haus. In: McE, S. 48-58 (Sigle „MH“).

13 Im Rauchsalon. In: McE, S. 73-88. (Sigle „Ra“); Ein Wrack. In: McE, S. $89-97$ (Sigle „Wr“); Eisenbahnerlebnisse in Amerika. In: McE, S. 98-113 (Sigle: EA); Jack Strap und der Drummer. In: McE, S. 114-121 (Sigle „JS“); Eine Minute mit Edison. In: McE, S. 122-135 (Sigle „ME“); Ein Seebad für Millionäre. In: McE, S. 136-151 (Sigle „SM“); Sammy und Dinah. In: McE, S. 152-164 (Sigle „SD“); Erinnerungen an Chicago. In: McE, S. 165-179 (Sigle „EC“); Aus Kentucky. In: McE, S. 180-209 (Sigle „Ke“); Kikapu. In: McE, S. 210-225 (Sigle „Ki“); Bei den Mormonen. In: McE, S. 226-241 (Sigle „BM“); Pike’s Peak. In: McE, S. 242-255 (Sigle „PP“); Ein Detective in San Francisco. In: McE, S. 256-273 (Sigle „SF“); Nach Klondyke. In: McE, S. 274-287 (Sigle „NK“).

14 Ein Pfingstritt durch den Peloponnes. In: McE, S. 288-304 (Sigle „Pe“); Eine Fahrt nach dem Heiligen Berg. In: McE, S. 305-327 (Sigle „HB“); Jostedalsbrä. In: McE, S. 328-342 (Sigle „Jo“); Wanderungen in Jotunheim. In: McE, S. 343-359 (Sigle „WJ“); Wie man das Abstürzen lernt. In: McE, S. 360-372 (Sigle „WA“).

15 Mulot-Déri: Alte ungenannte Tage, S. 297.

16 Goldbaum, Wilhelm: Ludwig Hevesi und Mac Eck. In: Pester Lloyd. 21.11.1900, Morgenblatt, o.S. 
17 Ebd. Auch Schnitzlers Tagebucheinträge verstärken diesen Eindruck. „Im Kfh. Eckstein; Fülle des Wissens, keine Persönlichkeit“ - notiert er am 7. Dezember 1894. Schnitzler: Tagebuch 1893-1902, S. 103.

18 - dm -: ,Mac Hevesi“. „,Mac Ecks sonderbare Reisen zwischen Constantinopel und San Francisco". Von Ludwig Hevesi. In: Wiener Zeitung, 05.12.1900, S. 8. Allen Ernstes erwägt der Rezensent auch die Möglichkeit einer Übersetzung aus dem Englischen. Ebd.

19 Goldbaum: Ludwig Hevesi und Mac Eck, S. 2.

20 Im Extremfall entfällt selbst dieser Hinweis. Vgl. SM 136.

21 Um nur zwei Beispiele für eine solche Zusatzinformation zu nennen: „Friedrich Eckstein, von dessen europäischen und amerikanischen Erlebnissen der Leser schon so manches Interessante in meiner Niederschrift gelesen hat." L. H-i.: Ein mystischer Besuch. Nach Mittheilungen eines Wieners. In: Fremden-Blatt, 25.12.1898, S. 11-13, hier S. 11; „Der Erzähler ist Friedrich Eckstein, dem unsere Leser bereits nach Amerika und auf den Berg Athos gefolgt sind; Julius ist sein Reisegefährte, der Architekt J. Mayreder." H.: Ein Pfingstritt durch den Peloponnes. Nach mündlichen Mittheilungen eines Wieners. In: Fremden-Blatt, 29.05.1898, S. 17-19, hier S. 17. Im Band übernimmt das Vorwort diese Funktion für alle Beiträge.

22 Vgl. noch FG 62; SD 162; Ki 223; BM 231; PP 242; PP 246; SF 261; Pe 297; HB 308; WJ 355.

23 „Im folgenden ist der Versuch gemacht, eine der größten Gletscherwanderungen [...] zu rekonstruieren. Sie wurde am 22. Juli 1886 angetreten. Die Wanderer waren mein Freund Friedrich Eckstein und sein Freund Aichinger. [...] Die Grundlage dieser Erzählung sind die Notizbücher Ecksteins und seine mündlichen Ergänzungen.“ Jo 328-329.

24 Vgl. auch: ,[...] antwortete Freund Fritz, als ich ihn auf mormonische Erlebnisse zu sondieren begann”. BM 226. Vgl. ebd. S. 227.

25 Vgl. EC 166.

26 Die englischen Wörter sind im Original - abhebend von der Frakturschrift - in lateinischen Lettern gedruckt. Hier werden sie kursiv wiedergegeben.

27 Vgl. Ki 214.

28 Mitteilungen (wenngleich nicht Nacherzählung) der Reiseerlebnisse anderer (vor allem in Übersee) begegnet man in den Tageszeitungen übrigens immer wieder. Vgl. z.B. N.N.: Aus der amerikanischen Gesellschaft. In: Fremden-Blatt. 22.03.1883, S. 11-12, hier S. 11: „Ein Stuttgarter Doktor, der sich zur Zeit in Amerika aufhält, gibt in einem Briefe an den »Sch. M.« folgendes anziehendes Bild aus der amerikanischen Gesellschaft: [...]".

29 „Einer der Passagiere erzählte nichts anderes als Anekdoten seines Freundes Travers [...]. Er ahmte ihn sogar körperlich nach“ (Ra 82) - berichtet „Fritz“. Das liest sich wiederum als metafiktionaler Hinweis auf die Beziehung Eckstein-Hevesi.

30 Vgl. Hevesi, Ludwig: Zerline Gabillon. Ein Künstlerleben. Stuttgart: Bonz 1894; Ders.: Ludwig Speidel. Eine literarisch-biographische Würdigung. Berlin: Meyer \& Jessen 1910.

31 Vgl. Brenner, Peter J.: Der Reisebericht in der deutschen Literatur: Ein Forschungsüberblick als Vorstudie zu einer Gattungsgeschichte. Tübingen: de Gruyter 1990 (=Internationales Archiv für Sozialgeschichte der deutschen Literatur, Sonderheft 2), S. 491-505.

32 Vgl. Schmidt, Alexander: Reisen in die Moderne. Der Amerika-Diskurs des deutschen Bürgertums vor dem Ersten Weltkrieg im europäischen Vergleich. Berlin: Akademie Verlag 1997, S. 67 ;

33 Vgl. ebd., S. 74.

34 Vgl. EA 98-100; Ki 225.

35 EA 103.

36 Vgl. ME 134-135. Die mit Th. A. Edison verbrachte eine Minute ist sogar in Ecksteins Autobiografie eingegangen. Eckstein: Alte unnenbare Tage, S. 265-268; Vgl. EA 104; SM 140; EC 173; Ke 183; Ke 188; Ke 193. 
37 Ra 74; EC 166; Ke 183-184; Ke 197; Ke 199; BM 227; BM 235; PP 242; PP 253.

38 Vgl. FG 65; ME 130; SD 153; EC 168; Ke 184; Ke 203; Ki 221; BM 229; PP 243; PP 245; PP 255; NK 274. Es handelt sich eher um flüchtige Beobachtungen als systematische Vergleiche.

39 „Wir räsonnierten dann noch weidlich über die Barschheit unseres Kapitäns, den wir für die Gelegenheit [Treffen mit einem Schiff unter Notflagge, E. H.] etwas kontinentaler, d.h. sentimentaler gewünscht hätten.” Wr 95; Eckstein reist mit der SS City of Paris. Wr 89.

40 „Sechsnullenmenschen“, SM 136; „Sechsmillenjüngling“, SM 145. Vgl. auch SM 137 sowie die Traumgeschichte des deutschen Einwanderers John Beck, BM 239.

$41 \mathrm{Vgl}$. auch die Anekdote von den aus Europa (mit Profit) eingeschifften historischen Dachziegeln. SM 141.

42 „ein ganz absonderliches Stück Stadtansicht“ [das Temple Square in Salt Lake City, E. H.], BM 231; Vgl. Ke 182 über die „Himmelkratzer“. Hierzu auch Hesse-Wartegg, Ernst: Himmelkratzer. In: Chicago. Eine Weltstadt im amerikanischen Westen. Stuttgart/Berlin/Leipzig: Union Deutsche Verlagsgesellschaft 1893, S. 75-90.

43 „Ueberhaupt erstaunt der Europäer immer wieder, wie viele fliegende Menschen er in Amerika sieht. Menschen werden dort so ohne Umstände zu Fenstern und Thüren hinaus-, oder von Brüstungen, Treppen, Dächern hinabgeworfen, als wenn sie gar nichts Gebrechliches wären.“ EC 168.

44 Gemeint ist eine Art Unkonventionalität: ,in Hemdärmeln, die Stiefel in die Höhe, den Hut auf dem Kopfe”, Ke 187. Vgl. auch „Ein Kentucky-Farmer!”, Ke 207); „Lynchungen“, Ke 190. Eckstein nennt aber auch andere Regionalklischees, wie etwa: „Eine Bostonerin ist ein geborener Professor und jede Dame dort wird als Fachmann in irgend etwas erzogen." PP 247.

45 BM $226 \mathrm{ff}$.

46 „Maple-room bedeutet eigentlich das Ahornzimmer, nämlich eine mit lichtem Naturholz getäfelte Trinkstube.“ SF 258.

47 , [I]n Elektrisch-Amerika giebt es keine Kerzen, sie gelten für »zu gefährlich«““ BM 236. Allerdings sorgt dieser Sachverhalt an der zitierten Stelle für die durch Stromausfall bedingte komplette Verfinsterung eines Hotels.

48 Um nur einige Bespiele zu nennen: EA; ME 128; ME 130; EC 172; EC 178.

49 Vgl. „Tennisheldenthaten“, SM 146; „Bicycle-Rennen“, EC 178.

50 Vgl. EA 105-106. Generell verbinden sich mit Zügen physikalisch-körperliche bzw. tumultuöse Szenen, vgl. EA 101; EA 102; FG 66ff; über diesen „Bankfeiertag“ vgl. auch Eckstein: „Alte unnennbare Tage", S. 262-264.

51 „Er lachte mich aus; da sei undenkbar, er glaube nicht, daß einer sich da hinausarbeiten könne [...]. [I]n sechs Minuten war ich oben." Ke 204.

52 Vgl. EC 166; Ke 182. 